\title{
Analysis Characteristics of Treating Chronic Prostatitis with External Therapy of Chinese Medicine
}

\author{
Mengfan Peng ${ }^{1, a}$, Baosong Liu ${ }^{1, b}$ and Mingsan Miao, \\ ${ }^{1}$ School of Pharmacy, Henan University of Chinese Medicine, Zhengzhou, Henan, China \\ apengmengfan@139.com, bliubaosong647@163.com, ${ }^{c}$ miaomingsan@163.com \\ *corresponding author
}

Keywords: Chronic Prostatitis, Etiology and Pathogenesis, Characteristics Analysis, External Therapy

\begin{abstract}
Chronic prostatitis (CP) is one of the most common diseases of adult males and urology, which affects the physical and mental health of patients severely. Currently the treatment of chronic prostatitis has entered comprehensive treatment era. External therapy plays an important position in comprehensive treatment because of the advantages as easy to use and better clinical curative effect. External therapies commonly include sitz bath, physical therapy, rectally, navel therapy, transuurethral perfusion, prostate injection and transperineal ultrasound. This article analyzes the Chinese medicine symptoms, etiology and pathogenesis, and characteristics of $\mathrm{CP}$ with external therapy of Chinese medicine.
\end{abstract}

\section{Introduction}

The causes of chronic prostatitis are complicated, the pathogenesis is unclear, and the clinical manifestations are diverse, seriously affect the patient's normal life. Chronic prostatitis is characterized by abnormal urination and pain in the surrounding area of the prostate, as well as more and more obvious mental disorders and individual specific clinical manifestations. Chemotherapeutic treatment has the disadvantage of side effects, and the patient is susceptible to tolerance and resistance. And traditional Chinese medicine external treatment is guided by the treatment of syndrome differentiation and the overall view, and through different ways such as orifice, skin and acupuncture points to achieve the goal of preventing and controlling diseases. In addition, the external treatment of traditional Chinese medicine has the advantages of flexible and diverse methods, large drug combination toughness, low side effect and good compliance. Therefore, compared with western medicine, traditional Chinese medicine is more easily accepted by patients. In this paper, we review the etiology, pathogenesis and characteristics of Chinese medicine for prostatitis, so as to promote the treatment of chronic prostatitis.

\section{Etiology and pathogenesis}

Chronic prostatitis is one of the most common diseases in the urology department. It is characterized by complicated pathogenesis, delay of course, multiple complications and recurrent attacks. Modern medicine believes that chronic prostatitis involves a variety of pathogenic factors including bacterial infection, urine reflux, autoimmune reaction, trace element deficiency and mental psychology [1]. Prostatitis belongs to the category of "gonorrhea", "white turbidity" and "white wholeness" in traditional Chinese medicine, and it is believed that blood stasis runs through the whole disease, and the local blood stasis is the key pathogenesis. Traditional Chinese medicine thinks that the main cause of this disease is wet and heat exhaustion, and it is the main cause of prostatitis. The three main causes of prostatitis are heat, exhaustion and kidney deficiency. Chronic prostatitis disease machine is the deficiency and the standard, the deficiency is kidney essence deficient, the heart and spleen two deficiency, the standard is the dampness and heat turbidity stasis [2,3]. 


\section{Syndrome differentiation of TCM external treatment of chronic prostatitis}

Chronic prostatitis is a common disease and multiple pathogenesis. It often involves many viscera and multiple systems, and the clinical symptoms are complicated and changeable. Chronic prostatitis disease machine is the deficiency and the standard. In the early stage, there are many evildoers, which are dampness and heat, qi stagnation, blood stasis, congealing, and long illness causes body deficiency. There are four common clinical classification about chronic prostatitis. It is shown in table 1 for details of the dampness and heat accumulation syndrome, qi stagnation and blood stasis syndrome, stasis and heat injury Yin syndrome, and kidney deficiency and blood stasis syndrome [4].

Treatment based on syndrome differentiation is the essence of traditional Chinese medicine, but different diseases have different pathological process, different characteristics of the drug, and pure disease differentiation is difficult to reflect the advantages of traditional Chinese medicine and individualized treatment, only the combination of disease and syndrome differentiation, can maximize curative effect. Chronic prostatitis is a clinical syndrome with different etiologies, different clinical manifestations, different disease processes and different treatment responses [5]. In the actual incidence with the deficiency and the standard, the single syndrome type is few. Therefore, the treatment of this disease should use different treatment methods, such as reinforcing kidney and strengthening resistance, eliminating dampness and heat, invigorated qi and energy, breaking the blood and relieving the pain. See table 1 for details.

Table 1. Common clinical classification of Chronic prostatitis

\begin{tabular}{|c|c|}
\hline Classification & Syndrome \\
\hline $\begin{array}{l}\text { Dampness and heat } \\
\text { accumulation }\end{array}$ & $\begin{array}{l}\text { Heat of the urethra, whitening of the urine, wet of the scrotum, tongue red, moss yellow or } \\
\text { greasy, pulse slip }\end{array}$ \\
\hline Qi stagnation and blood stasis & The pain is primarily, the tongue is dark, or has petechia, pulse string or astringent \\
\hline Stasis and heat injury Yin & $\begin{array}{l}\text { Urgency, frequent urination, heat of urethra, premature ejaculation, tongue red or crack, } \\
\text { moss less or no moss, pulse fine }\end{array}$ \\
\hline $\begin{array}{l}\text { Kidney deficiency and blood } \\
\text { stasis }\end{array}$ & $\begin{array}{l}\text { Kidney qi deficiency is primarily, the urine is not infrequent and has the residual, the } \\
\text { slippery essence premature ejaculation, tongue light or fat big partial dark, the mossy white } \\
\text { or thin, pulse string thin }\end{array}$ \\
\hline
\end{tabular}

\section{TCM external therapy for chronic prostatitis}

Ancient records "The principle of external treatment is same as internal treatment". External treatment of Chinese medicine is an important part of Chinese medicine, which is paid equal attention to internal treatment. External treatment of Chinese medicine has a long history of treatment. Compared with the internal treatment, external treatment has the advantages of high concentration of local drugs, quick response and less adverse reactions. There are acupuncture treatment, acupoint application, traditional Chinese medicine sitting bath, umbilical therapy, urethral perfusion, Chinese medicine rectal administration and so on [6].

\section{A. Acupuncture therapy}

Acupuncture can dilate blood vessels and lymphatic vessels, increase excited point sensors, affect organizational endocrine environment, adjust the body's humoral immune function, to reduce the swelling anti-inflammatory, promoting repair, adjust the secretion and other functions [8]. Stir-poisonous moxibustion is originated from the Ming dynasty combined TCM internal medicine, in treating downward flow of damp-heat syndrome, liver depression and qi stagnation syndrome and Yin deficiency fire excess III type prostatitis patients achieved good therapeutic effect [9]. The treatment of chronic prostatitis was treated by acupuncture mainly in the middle, taichong, perineum and taixi. The scores of patients with chronic prostatic inflammatory index and depression self-test scale were lower than those in the control group. Symptoms such as difficulty in urination, pain, and sexual dysfunction were 
significantly alleviated, with low recurrence rate and good prognosis, which could significantly improve the patient's bad mood [10]. The local blood circulation of prostate can be improved by the rank edge and middle pole cavity, guan yuan, middle pole, qi hai cave belong to ren mai combined shenshu point can enrich kidney essence, warm cold dampness. The above acupoints were used to treat chronic prostatitis of kidney Yang deficiency, and the total effective rate was 92.5\% [11].

\section{B. Acupoint application therapy}

The external application of traditional Chinese medicine can be administered locally, to achieve the effect of invigorating blood circulation, reducing swelling and relieving pain, warming kidneys inducing diuresis for treating stranguria. Shuangbai Powder is composed of zellan, rhubarb, cacumen biotae, cypress, etc., which has the effect of clearing heat and promoting diuresis, promoting blood circulation to remove blood stasis. Under the patient's lower abdomen hot compress Shuangbai Powder, beneficial to adjacent prostate organ acupoint to absorb. It is effective to treat chronic prostatitis with Shuangbai Powder and light irradiation. The total effective rate is $96.88 \%$, and it is non-toxic side effect and convenient for drug administration [11]. The Shenque acupoint is closely related to ren, governor, chong, and the veins, which are all meridians of the whole body. Drugs can be used through the Shenque acupoint and all the meridians of the body to achieve therapeutic. Make clearing heat and promoting diuresisas, tonifying kidney and detoxifying blood as treatment principle, in Shenque acupoint and Guanyuan acupuncture external application Qianleningshan can obviously improve the abnormal symptoms of urine, relieve the inflammation of the prostate gland, and promote the recovery of prostate gland function [12].

\section{Traditional Chinese medicine sitting bath}

Traditional Chinese medicine bath can be applied to the surrounding area of the prostate by the dual temperature of medicine and physics. Moreover, the effective components of traditional Chinese medicine can penetrate into the prostate directly through the skin mucosa of perineum, anus and the rectum, lymph nodes, blood vessels, etc. In turn, the local blood circulation of the prostate is increased, and the absorption and dissipation of inflammation can be promoted to reduce the clinical symptoms of the prostate. A randomized controlled clinical trial study showed that Tongqianluotang sitting bath fumigation on the improvement of symptoms in patients with chronic prostatitis with qi stagnation and blood stasis was positive, and the efficiency was significantly higher than that of the control group [13]. Other studies have shown that, Mongolian medicine sitting bath combined massage to treat the acute and chronic prostatitis in a short course of treatment is good, the inflammation is absorbed quickly, the recurrence rate is low, and the normal function of the gland can be restored and the course of treatment can be shortened [14].

\section{Other therapies}

Ligustrazine can significantly reduce the content of oxygen free radicals, histamine, prostaglandin E, and 5-hydroxytryptamine in inflammatory exudates. Ligustrazine through ultrasonic conductivity prostatic therapy apparatus rectal administration can effectively improve clinical symptoms of type III chronic prostatitis patients, and the curative effect is stable [15]. Research shows that urethral perfusion can not only treat the potential inflammation of the urethra, but also cure prostatitis [16]. A multi-center, large-

sample, randomized, double-blind controlled clinical study that lasted for about 5 years showed that Ding Gui scattered apply umbilical united Qianlieanshuan returning anus to treat type III chronic prostatitis patients has good therapeutic effect and safety [17].

\section{Characteristics analysisChronic prostatitis external treatment of traditional Chinese medicine} Combined with the literature, the characteristics of external therapy for prostatitis are: the combination of traditional Chinese medicine internal and external use, the combination of Chinese medicine and western 
medicine, the combination of TCM external therapy and physical therapy, and the comprehensive application of various external therapies. Prostatitis is a common disease of adult male, with long course of disease and complex etiology. It is involved in pathological abnormalities and different types of complications of heart, liver, spleen, kidney, bladder and other viscera functions, single herb or single therapy cannot cure the disease completely.

The external application of traditional Chinese medicine can be administered locally, to achieve the effect of invigorating blood circulation, reducing swelling and relieving pain, warming kidneys inducing diuresis for treating stranguria. Through external use and internal of traditional Chinese medicine, it can achieve the TCM characteristics and advantages of holistic syndrome differentiation, internal and external treatment, and improve the clinical efficacy, reduce the recurrence rate and have a good prognosis. Modern medicine commonly use quinolones antibacterial drugs, non-steroidal antiinflammatory drugs, alpha receptor antagonists, $\mathrm{M}$ receptor antagonists, surgery, etc. to treat chronic prostatitis. But a prolonged treatment found that most drugs could lead to damage of liver, kidney, stomach, liver, kidney or itself has toxicity, while under the effect of Chinese medicine external treatment intervention, not only improve the curative effect and the various types of adverse reactions are improved. Between different external therapy of traditional Chinese medicine does not define the particular combination, but on the basis of syndrome differentiation to harmony the body's viscera and immune regulating function of patients with chronic prostatitis, avoiding the first overdose of oral drugs and stimulation of the gastrointestinal tract, which is the most acceptable treatment for patients. Massage is the most traditional physical therapy to promote the emptying of the prostate tube; Heat therapy is a kind of thermal effect that uses various physical means to increase the blood circulation of prostate tissue, accelerate metabolism, eliminate tissue edema, and relieve pelvic floor muscle spasm. These physiotherapy can accelerate the absorption of external drugs and improve the local blood concentration. To sum up, the combination of traditional Chinese medicine external therapies and other therapies, and the combination of various external therapies can take into consideration various factors, and can treat prostatitis from the overall perspective.

\section{Outlook}

External treatment of Chinese medicine is an important part of Chinese medicine, which is paid equal attention to internal treatment. The etiology and pathogenesis of prostatitis is complicated, and involves various viscera functions such as heart, liver, spleen, kidney and bladder. The research achievements in TCM syndrome distribution, theoretical innovation, clinical efficacy evaluation and intervention mechanism of TCM in chronic prostatitis have reached a preliminary consensus in the industry [18]. The external treatment of prostatitis is flexible and varied, so that people can get out of traditional Chinese medicine. The external therapy of traditional Chinese medicine can be combined with each other, and also can be combined with western medicine or physical therapy, thus to broaden the treatment thoughts of prostatitis.

But Chinese medicine external treatment clinical research more contrast with the western medicine treatment group, lacking comparative study of patients with no serious drug interference, which is a disadvantage in the evaluation of the curative effect of the treatment of prostatitis. Chinese medicine external treatment is often associated with western medicine for the treatment of chronic prostatitis, influence the efficacy and safety of traditional Chinese medicine external treatment of prostatitis. The etiology and pathogenesis of prostatitis are complex and the clinical symptoms are diverse. It is inevitable that there will be errors in the grouping of patients, which will affect the evaluation of external therapeutic effect. Sitting bath, fumigation, warm acupuncture and ionic introduction can be controlled by improper temperature, resulting in skin burns and difficult to maintain treatment. Acupuncture treatment 
can lead to infection in the pinhole, and its pain can aggravate the sensitivity of the patients with prostatitis and reject treatment. Ointments and excipients are used for local skin, which can cause itching, redness and allergic reactions.

The treatment of prostatitis with traditional Chinese medicine has unique advantages, although it has disadvantages, it is not to be abandoned. For this purpose, non-drug and other physical therapy intervention groups should be adopted in clinical studies to strengthen the basic, normative, systematic and in-depth clinical research. Emphatically from the theoretical mechanism of the treatment, the treatment design, operation methods, drug compatibility, excipients, etc to improve the effect of external treatment of prostatitis, making external treatment of traditional Chinese medicines into reasonable prostatitis specification standard. Although the integrated application of many kinds of therapy can achieve a series of effects, but any therapy has no absolute safety. So we should try to minimize the various treatment methods and drug concentration in the treatment of the same patients, to find reasonable effect of external treatment methods with the exact drugs to reduce the potential threat. To sum up, the external therapy of prostatitis has potentially huge application value, which is worthy of clinical promotion and further study.

\section{Acknowledgment}

This research was financially supported by the National "11th Five-Year" support program (2008BAI53B09), Standardization of traditional Chinese Medicine of State Administration of traditional Chinese medicine (2017-149-11).

\section{References}

[1] Shun Zhixue, Li Pengchao. Discuss Chronic prostatitis based on the pathogenesis of deficiency and the standard [J]. New Traditional Chinese Medicine. 2017, 49 (07): 183-184.

[2] Zheng Xiaoting, Chen Dong, Yin Shen. Progress in the pathogenesis of chronic prostatitis [J]. Chinese Journal of Traditional Chinese Medicine. 2016, 34 (02): 286-288.

[3] Chen Rui, Zhang Yaosheng. A brief discussion on the etiology and pathogenesis of prostatitis [J]. Chinese Medicine Guide. 2012, 10 (34): 285-286.

[4] You Xujun, Yuan Shaoying. Yuan shaoying's experience in treating chronic prostatitis [J]. New Traditional Chinese Medicine. 2017, 49 (04): 174-176.

[5] Mo Xuwei, Wang Shan, Li Haisong. Progress in the treatment of chronic prostatitis [J]. Universal Chinese Medicine. 2015, 8 (07): 878-883.

[6] Wang Shengbo, Lu Zhijie. Progress in the research of TCM external therapy for chronic prostatitis [J]. Journal of Nanjing University of Chinese Medicine. 2014, 30 (03): 297-300.

[7] Wang Yajie, Dai Ning. Study on the mechanism and status of chronic prostatitis by acupuncture and moxibustion [J]. Clinical Journal of Traditional Chinese Medicine. 2014, 26 (01): 107-108.

[8] Chen Qi, Li Qiang, Huang Xiuqin. Stir-poisonous moxibustion pull out poison acupuncture combined Chinese medicine treat type III prostatitis 3cases [J]. The World's Newest Medical Information Digest. 2017, (58): 154+158.

[9] Yang Wubin. The therapeutic effect of acupuncture on patients with chronic prostatitis [J]. Chinese Medical Science. 2017, 7 (01): 70-73.

[10] Ma Guizhi, Chen Lei, Feng Xinxin. Therapeutic effect of warm acupuncture on chronic prostatitis of kidney-yang deficiency [J]. Shanghai Acupuncture Journal. 2016, 35 (07): 820-822.

[11] Han Zhaohong, Li Baozhu, Feng Xianlan. The clinical observation and nursing of the treatment of chronic prostatitis by using Shuangbai powder and combination with divine lamp [J]. Chinese General Nursing. 2013, 11 (09): 781-782. 
[12] Xu Zejie, Zheng Wenhua, Li Xizhu. The treatment of 100 cases of chronic bacterial prostatitis treated by the combination of Qianleningshan and special traditional Chinese medicine external treatment [J]. The Traditional Chinese Medicine in Hebei. 2015, 37 (11): 1616-1619.

[13] Li Duoduo, Ma Wei, Wang Bin. Tongqianluotang sitting bath fumigation to treat 52 cases of chronic prostatitis with qi stagnation and blood stasis [J]. Chinese Journal of Clinical Medicine. 2015, 43 (12): 40-43.

[14] Huo·Daoerjiala, QiaoLongbate. Mongolian medicine sitting bath combined massage to treat prostatitis [J]. Chinese Journal of National Medicine. 2013, 19 (10): 48.

[15] Wang Bo, Shi Benkang. Therapeutic effect observation of Ligustrazine through ultrasonic conductivity prostatic therapy apparatus rectal administrationon type III chronic prostatitis patients [J]. Shandong Medicine. 2017, 57 (02): 85-86.

[16] He Mingwei, Gao Jiangyuan, Luan Xingyu. The effect of urethral perfusion on chronic prostatitis [J]. Hebei Medical. 2012, 18 (06): 751-754.

[17] Li Haisong, Wangbin, Han Liang. Clinical study on 80 cases of III type prostatitis (Qi stagnation blood stasis typet) reated Umbilical therapy combined combined Suppository [J]. Journal of Beijing University of Chinese Medicine (Clinical edition). 2013, 20 (02): 19-23.

[18] Li Haisong, Han Liang, Wangbin. Progress and thinking of TCM research on chronic prostatitis [J]. Universal Chinese Medicine. 2012, 5 (07): 481-484. 